\title{
THE BLOOD SUPPLY OF NERVES
}

\author{
By ARchibald Durward, F.R.S.(Ed.), M.D., M.B., Ch.B. \\ Professor of Anatomy, University of Leeds.
}

Considerable attention has been given to the blood supply of nerves in the past few years. This does not imply that nothing was previously known ; Adams (1942) reviewed the literature on the blood supply of nerves and brought together the widely scattered and surprisingly extensive information on that topic. Much of the present-day interest naturally derives from the great numbers of peripheral nerve casualties which have arisen in the war. A more detailed conception of the whole pattern of the vascularization of peripheral nervous tissues is now available and a good deal of experimental evidence in this field has accumulated.

There undoubtedly has been some kind of assumption that the blood supply of nerves was of no great moment. Peripheral nerves have been treated surgically with a total lack of respect for the arteries or the veins associated with them. Similar treatment has not been accorded to the gut or the central nervous system. That extensive operations can be, and commonly are, successfully carried out on peripheral nerves, with little or no thought as to their blood supply, strongly suggests that the pattern of vessels in nerves is peculiar and is so disposed as to allow extensive mobilization without essential devascularization. The surgeon has long acted, maybe unwittingly, on this assumption. This paper will be concerned with a review of our knowledge regarding the blood supply of nerves and of recent experimental work in this field.

\section{Nutrient Arteries of Nerves}

There are no major arteries specifically devoted to the supply of nerves. Each nerve, large or small, receives nutrient twigs from adjacent arteries. Such nutrient vessels are gerierally short, and although their size is variable, many of them may be seen with the naked eye. These are local sources of supply presenting as much variation in actual position as do vessels elsewhere. Some are more or less predictable in position, e.g., at the elbow in the case of the ulnar nerve, and in the spiral groove in the case of the radial nerve (Sunderland, I945), but from case to case a good deal of variation may be found, and variations on the two sides of the body are not uncommon. But one would stress that this does not alter the essential plan, namely, that each nerve receives along its course a variety of nutrient arteries coming from adjacent vessels. It is true that in certain positions more sizable arteries are occasionally found associated with nerves, e.g., the sciatic and median, and such vessels running on the nerve for considerable distances are to be regarded rather as embryological persistences and do not imply that the nerves concerned are receiving any preferential vascular supply.

A detailed and lucid account of the anatomy of the nutrient arteries to the nerves of upper limb and to certain rerves of the lower limb has recently been given by Sunderland (1945, a and c).

\section{Epineural Vessels}

On reaching the nerve, these small local nutrien arteries generally divide into ascending and de - . scending branches which course in the epineurium. There may be a number of such epineural vessels, often visible to the naked eye. Sometimes such vessels pass deeply into the nerve and run between the fasciculi. From these primary divisions of the nutrient artery secondary branches are given off penetrating more deeply and dividing further. The vessels concerned are arranged predominantly in a longitudinal fashion in the perineural or interfasicular connective tissues. Finally, by repeated branchings, the finest vessels penetrate within the fasciculi and form there a capillary bed, again arranged mainly in a longitudinal fashion with numerous cross connections. Vessels of precapillary size may be found at times within the actual fasciculi but most generally the intrafascicular vessels are of the capillary order.

\section{The Intrafascicular Plexus}

This intrafascicular plexus is common to all the nutrient arteries and is continuous throughout the length of the nerve. It is fed, or reinforced at various levels, by the nutrient arteries, but no one nutrient artery may be considered as dominating any portion of the plexus. The arrangement outlined above is virtually the antithesis of the 'end artery' conception : it is an arrangement whereby a rich continuous capillary bed occupies the length 
of the nerve and receives regional reinforcements here and there.

The view that the circulation within the nerve might be dominated ir particular segments by the local nutrient artery has in the past been put forward, and, were it a fact, would be of considerable surgical importance. The extensive mobilizations of nerves carried out in operations scarcely lend support to the view, and certain experimental findings in animals very strongly suggest that the role of any particular nutrient artery in the supply of its own region of the nerve is virtually nonessential. In this connection Adams (1943) reported that in the rabbit's sciatic nerve the destruction of the nutrient arteries in the thigh led to negligible degenerative changes in the majority of the animals investigated; he had, however, a small minority of animals in which quite extensive degenerations occurred and this minority, though small, left open a doubt as to whether there might be some significant variation in the supply of the nerve in the cases concerned which might have led to the dominance of one nutrient artery, or, again, as to whether there might not be some other factor coming into play to give the discordant result.

\section{Experimental Study of Blood Supply}

The writer has investigated the problem from two angles in the rabbit's sciatic nerve.

(a) In one series of animals the nerve was mobilized completely along its course from the buttock to the knee and all nutrient arteries approaching the nerve severed. In addition the obvious epineural vessels were severed so as to interrupt, as far as possible, the longitudinal system of vessels. The latter procedure, carried out under a dissecting microscope, did not, of course, affect any vessels running within the nerve and could not, therefore, obliterate more than a proportion of the larger epineural vessels. The results indicated that following on destruction of the epineural vessels there was invariably some degeneration, varying from case to case, but never massive, and, in comparison with Adam's results (excluding his discrepant findings) the degeneration induced was of a greater order. The escape of the nerve from any obvious degeneration following on the mere ligation of the nutrient vessels and the invariable production of degeneration on the destruction of epineural vessels, very strongly suggests that the latter are in some way more vital than the former. It is suggested that by destruction of the epineural vessels some portions of the longitudinal capillary bed served by such vessels would be put out of action and the resulting degeneration explained on such grounds. In this connection it seems not unlikely that the dis- cordant results obtained by Adams might readi be explained either by destruction of, or damag done to, epineural vessels during manipulation, of perhaps, more likely, to thrombosis within the ligated vessels spreading into the epineural vesse or beyond and so involving the longitudinat vascular pathway within the nerve. This sugo gestion, though speculative, is supported b $\overline{\bar{p}}$ Sunderland ( 1945, b) who states : "If the nerve roughly and carelessly stripped from its be these delicate channels are likely to be torn .. interrupting thereby the superficial system on the surface of the nerve, a disturbance which, in turn, is liable to embarrass the intraneuraf circulation.'

(b) In another series of rabbits the sciatie. nerve, after mobilization in the thigh and ligation of all nutrient arteries, was ligatured at the leveb of the knee. This left the sciatic nerve completefo devoid of any nutrient supply along its course in the thigh and also it ensured the complete oblitera tion of the longitudinal vascular bed within the nerve at the site of the ligature. Previous experiments (e.g., Adams, r943) had made it clear thä the mere ligation of the nutrient vessels did no necessarily lead to any extensive degeneration within the nerve. The object of this serieso experiments was to take the nerve with ies regional sources obliterated and then, in addition embarrass the intraneural circulation by preventing the free flow of blood throughout the length of the segment under observation. The animals wers allowed to survive for ten days and nerves in the thigh were then examined, either histologically ou by injection. Those subjected to histology showeg that there was invariably degeneration induced within the nerve. The degeneration was nevent. massive but it tended to be more extensive in the more distal parts of the nerve (the segment of the nerve immediately adjacent to the ligature was not considered in the assessment of the result). In those cases which were injected, the nerves were subsequently cleared and a comparison made between the injected experimental nerves ang injected normal nerves. It was found that the nerves which had been mobilized and ligature took the injection mass virtually as well as normab nerves except in the lowest segment adjacent to the ligature. These injections were carried ouf sufficiently long after the operation, to precludi any possibility of leakage from cut vessels, an suggested strongly that the vascular bed within the nerve did not suffer appreciably.

It may be concluded from the above experiments that though destruction of nutrient arteries apo proaching the nerve has little or no effect, destruco tion of the epineural vessels leads to limite 
degenerative changes depending on the extent of epineural vascular deprivation. The destruction of the epineural vessels, it is suggested, destroys in part the longitudinal vascular bed of the nerve and to that extent renders the region ischaemic. It is further evident that the arterial and venous circulation in a peripheral nerve is adequate to maintain an effective circulation, even when a complete through-circulation is denied, as by ligature of the nerve distally. These findings, of course, relate to the nerve involved in the experiments and it is quite conceivable that the longer lengths of nerve involved in human operations might vary the severity of such degenerative changes as occur. Seddon and Holmes (1945) have reported ischaemic damage in the peripheral stump of the divided median nerve in the upper third of the forearm. In the case concerned the anterior interosseous artery had been destroyed. It would be unusual for the median nerve not to receive nutrient vessels in its course through the arm (Sunderland, 1945, a) and were such vessels present and intact, the occurrence of an ischaemic lesion would be surprising; but there remains the ever-present possibility of variation in the individual, and the absence of a nutrient branch to the median nerve in its course through the arm, though unusual, has been recorded. With a severed nerve, it would be the lowest segment which would show ischaemia, and in the case quoted by Seddon and Holmes, the severance of the anterior interosseous artery in addition was doubtless the crucial factor in inducing the severe ischaemia.

\section{Obliteration of Intraneural Vessels by Pressure}

The effects of local pressure on the circulation within a peripheral nerve have been studied by various workers. There are inherent difficulties in all methods and the complete occlusion of the intraneural vascular bed by pressures which would avoid structural damage to nerve fibres, is a matter of difficulty, since the vessels lying upon and within the nerve are of varying sizes and, as has been pointed out previously, vessels of a considerable size may course amongst the fasciculi and so escape occlusion by pressures which would obliterate capillaries. There is, however, a body of evidence derived from observations on compression of nerves, and two references to such work may be made here. Lewis, Pickering and Rothschild (I93I) investigated the effects of the arrested blood flow to a limb in man, by means of clamps and encircling cuffs. They were primarily concerned in the study of the resulting motor and sensory deficits and the recovery therefrom, but their conclusions indicated very clearly that all the effects described were attributable to ischaemia $\stackrel{\Omega}{\propto}$ and not in any sense to compression of the nerve $c$ fibres. More recently, Denny Brown and Brenner $\Rightarrow$ (1944) have studied in experimental animals the $\stackrel{5}{?}$ effects of graduated pressures induced by spring음 clips. Complete histological examination was $\frac{\overline{0}}{\overline{0}}$ possible and they were able to observe the presence $\frac{\bar{c}}{\sigma}$ or absence of degenerative changes in the nerves. $\mathbb{Q}$ From our viewpoint, the interesting conclusion $\%$ was drawn 'that the effect of pressure on the $\vec{\circ}$ nerve is considered to be due entirely to ischaemia.'

These two sets of observations carried out by $\vec{\omega}$ very different methods - one in man and the other in the experimental animal-give the strongest $\frac{0}{3}$ support to the view that the intraneural vascular bed is vital in the normal functioning of the nerve.

If local pressures induced by clamps or other o mechanisms can induce sensory and motor dis- turbances, and even degenerative changes, by occlusion of the vascular bed within the nerve, $\Rightarrow$ it is obviously conceivable that vasospastic states involving nerves may do the same thing. This possibility has been investigated in the case of the 8th cranial nerve by Atkinson (194I), and following on his work, Adams and Robinson (194D) $\overrightarrow{0}$ investigated the possibility of trigeminal neuralga $+\infty$ being essentially a vasospastic condition. The exhibition of vasodilator drugs did lend sone support to the view that in certain cases at any rate vasospasm within the trigeminal nerve was at the root of the sensory disturbance. Whether $\frac{0}{D}$ neuralgias are to regarded as commonly due to, or $\cong$ only occasionally caused by, vasospasm, is a $\overrightarrow{\overrightarrow{0}}$ point on which no final decision may yet be given, $\exists$ but the experimental evidence suggests that such a mechanism is by no means unlikely.

From a different approach, the role of the $\frac{\overline{3}}{3}$ circulation within the nerve has been studied by Bacsich and Wyburn (1945). Their work was 3 . carried out on the rabbit's sciatic nerve and comparisons were made between the regeneration rate in a nerve with a normal blood supply and a 0 nerve deprived of its regional sources of supply. They have concluded that no significant change in $\frac{}{2}$ the regeneration rate of nerves is attributable to experimentally induced variations in blood supply $\Omega$ and this lends further support to the view that $N$ the essential vascular bed of the nerve is a con- N tinuous longitudinal system, not regionally de- 0 pendent on the nutrient arteries.

\section{Summary}

Anatomical. Each nerve receives local branches $T$ of supply from adjacent arteries. Though pre- $\frac{O}{\mathbb{D}}$ senting the customary variation in their arrange- $\cong$ ment, associated with small arteries in general, the $\stackrel{\mathbb{D}}{\stackrel{D}{\varrho}}$ 
position of such nutrient arteries is reasonably predictable.

On the nerve a series of epineural vessels is formed, whose branches pass into the perineurium and, finally, there is produced within the fasciculi a longitudinally disposed capillary plexus which is continuous throughout the length of the nerve and is not dominated by local nutrient vessels.

Experimental. The intraneural plexus forms a system which is normally reinforced by the local nutrient arteries, but which can function adequately in a given length of nerve even when the local branches are ligated.

Obliteration of epineural or intraneural vessels produces degeneration of varying degree depending on the extent of vascular deprivation.

The complete and intact system of vessels upon and more particularly within the nerve is function ally more important than any localized group of nutrient vessels.

\section{BIBLIOGRAPHY}

ADAMS, W. E. (1942), F. Anat., Lond., 76, 323

ADAMS, W. E. (1943), F. Anat., Lond., 77, 243.

ADAMS, W. E. and ROBINSON, W. (194I), Lancet, Novembe 8th, p. 555 .

ATKINSON, M. (1941), f. Amer. Med. Ass., 116, 1753.

BACSICH, P. and WYBURN, G. M. (1945), Ұ. Anat., 79, 74.

DENNY BROWN, D. and BRENNER, C. (1944), Arch. Neur. छी Psychiat., 52, I.

LEWIS, T., PICKERING, G. W. and ROTHSCHILD, P. (193 I $\vec{p}$ Heart, 16, I.

SEDDON, H. J. and HOLMES, W. (1945), Brit. F. Surg., 32, 38 SUNDERLAND, S. (1945), (a) Arch. Neur. E Psychiat., Chicaga 53,91 .

SUNDERLAND, S. (1945), (b) Arch. Neur. \& Psychiat., Chicag@ 54, 280.

SUNDERLAND. S. (1945), (c) Arch. Neur. $\bullet$ Psychiat., Chicag\% 54, 283 .

\title{
$\int$ \\ CONFUSIONAL STATES IN ACUTE DISEASE
}

\author{
By J. M. NaISh, M.D., M.R.C.P. \\ Tutor in Medicine, University of Bristol.
}

Many a patient's battle to overcome an acute disease is lost because, failing to stand the strain of the illness, he becomes delirious. The additional burden thrown upon his body by restlessness, fear, and failure to obey those trying to help, is sufficient to tip the balance against him. The problem which such cases present is a common one, and, lying in the no-man's-land between general medicine and psychiatry, does not receive the attention which it needs.

\section{Classical Delirium}

There is nowhere any sharp dividing line between the full-blown picture of severe delirium and the mild nocturnal confusion of thought so frequently found in ill people.

Many patients, particularly the elderly, become confused when their illness is at its height. This confusion and lack of grasp, always at its worst nocturnally, may lead them to get out of bed in the belief that they should be going out to work, or to put their shoes on beneath the sheets to keep their feet warm. They are often shamefaced in the morning and attribute their experiences to a bad dream.

Fully developed delirium leads to well-defined physical and mental changes in these patients, and these should be kept clearly in mind.
The physical manifestations are restlessness, in somnia, incontinence and anorexia ; examination usually shows some pyrexia and tachycardia, aD furred tongue and slurring of the speech.

The mental changes vary from one moment to the next. The changing form of the menta? picture is perhaps the most typical finding in delirium and is due to the extreme suggestibility of delirious people whose moods and behavious are swayed both by their physical surroundings and their attendants. The prevailing delirious mood, however, is one of fear and horror; their speech abounds with talk of struggles, crises ancis bankruptcy. Restless activity and complete ino somnia dominate the picture.

The delirious are not only prone to invent, or t命 elaborate the truth in a broken sort of way, but they misinterpret their present surroundings and instructions. Essentially, this inability to interpret or understand the present has the same roots failure of memory, concentration, and mentas grasp-as the tendency to invent the past. The can only do the simplest and shortest test problems because they cannot hold their brains to the taske? for more than a few moments at a time. It iso true to say, however, that some delirious patients. can, by a superhuman effort of will, achieve mentad clarity when circumstances urgently demand it. 